\title{
Über die angenäherte Auflösung linearer Gleichungen in ganzen Zahlen.
}

$$
\text { von }
$$
A. Khintchine (Saratow).

\section{1.}

Im folgenden bedeuten alle lateinischen Buchstaben ganze rationale alle griechischen - reelle Zahlen.

Für einen beliebigen Punkt $\Theta=\left(\Theta_{1}, \Theta_{2}, \ldots, \Theta_{n}\right)$ des $R^{n}$ und eine beliebige reelle $\mathrm{Zahl} \beta$ setze man

$$
\begin{aligned}
& \operatorname{Min}_{\mid x_{i}: \leqq \lambda, 1 \leqq i \leqq n}\left|\sum_{i=1}^{n} \theta_{i} x_{i}-y-\beta\right|=\mu_{1}(\theta, \beta, \lambda), \\
& \operatorname{Min}_{\substack{x_{r} \mid \leq i \ldots 1 \leq i \leq n \\
\Sigma x_{i}^{2}>0}}\left|\sum_{i=1}^{n} \Theta_{i} x_{i}-y\right|=\mu(\Theta, \lambda) \\
& \lim \sup _{l, \rightarrow \infty} \lambda^{n} \mu(\Theta, \beta, \lambda)=\Phi(\Theta, \beta) . \\
& \lim \inf _{\lambda \rightarrow \infty} \lambda^{n} \mu(\Theta, \beta, \lambda)=\varphi(\theta, \beta), \\
& \lim \sup _{\lambda \rightarrow \infty} \lambda^{n} \mu(\Theta, \lambda)=\Phi(\Theta), \\
& \lim \inf _{\lambda \rightarrow \infty} \lambda^{n} \mu(\theta, \lambda)=\varphi(\theta) \text {. }
\end{aligned}
$$

$\mathrm{Zu}$ den klassischen Ergebnissen gehört, dass sțets 


$$
0 \leqq \varphi(\Theta) \leqq \Phi(\Theta) \leqq 1
$$

$$
x_{1} \xi+x_{2} \eta_{1}-R=0
$$

ist $\left.{ }^{1}\right)$, während $\varphi(\Theta, \beta)$ und $\Phi(\Theta, \beta)$ im allgemeinen beliebige Werte zwischen 0 und $+\infty$ (beides einschliesslich) annehmen können ${ }^{2}$ ). Vor etwa 12 Jahren habe ich gezeigt ${ }^{3}$ ): Die Bedingung $\varphi(\theta)>0$ ist notwendig und hinreichend, damit $\Phi(\Theta, \beta)$ für jedes $\beta$ endlich sei. Hier werde ich zeigen; dass eine ganz ähnliche Beziehung zwischen $\Phi(\Theta)$ und $\varphi(\Theta, \beta)$ besteht es gilt nämlich der

SATZ: - Damit $\varphi(\Theta, \beta)$ für jedes $\dot{\beta}$ endlich sei, ist die Bedingung $\Phi(\Theta)>0$ notwendig und hinreichend.

Die Nummern 2.-3. sind dem Beweis dieser Behauptung gewidmet; in 4 , werden die zwischen den beiden Fragestellungen bestehenden Unterschiede besprochen. Nur der kürzeren Schreibweise halber ist überall $n=2$ angenommen.

$$
2 .
$$

HILFSSATZ 1, - Für $\Theta=\left(\Theta_{1}, \Theta_{2}\right)$ sei $\Phi(\Theta)=0$. Dann gibt es für

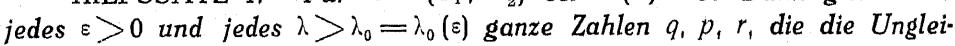
chungen

$$
0<q<\varepsilon \lambda^{2},\left|q \Theta_{1}-p\right|<\frac{1}{\lambda},\left|q \Theta_{2}-r\right|<\frac{1}{\lambda}
$$

erfüllen.

Beweis, - Wegen $\Phi(\Theta)=0$ lassen sich für jedes $\varepsilon>0$ und jedes $\lambda_{1}>\lambda_{0}=\lambda_{0}(\varepsilon)$ ganze Zahlen $x_{1}, x_{2}, y$ derart angeben, dass

$$
0<p=\sqrt{x_{1}^{2}+x_{2}^{2}}<\frac{\varepsilon}{1920} \sqrt{\lambda_{1}},\left|x_{1} \Theta_{1}+x_{2} \theta_{2}-y\right|<\frac{1}{\lambda_{1}}
$$

ist. Für jedes grosse $\lambda$ setze man $\lambda_{1}=96 \lambda^{2}, m=[192 \rho \lambda]$. Die Variable $q$ möge die Zahlen $0,1,2, \ldots, m$ durchlaufen, und man setze

Wegen (1) ist

$$
\xi_{q}=q \Theta_{1}-\left[q \Theta_{1}\right], \eta_{q}=q \Theta_{2}-\left[q \Theta_{2}\right] \text {. }
$$

$$
x_{1} \xi_{q}+x_{2} \eta_{q}=R+\frac{\delta q}{\lambda_{1}}
$$

wo $R$ ganz und $|\partial|<1$ ist. Jeder der Punkte $\alpha_{q j}\left(\xi_{q}, \eta_{q}\right)$ des Einheitsquadrats ist somit von einer der Geraden

1) Vgl, z. B. Koksma, Diophantische Approximationen (Ergebnisse der Mathematik und ihrer Grenzgebiete, Vierter Band, 4, Springer 1935; im folgenden als K, zitiert) Kap. I, Satz 1.

$\left.{ }^{2}\right)$ K, Kap, VII, Sațz 3.

8) Rec, Math. Sòc, Math, Móscou, 32, 203-219, 1924,

$\left(x_{1}, x_{2}\right.$ fest, $R$ ganzzahlig variabel) um weniger als

$$
\frac{q}{\rho \lambda_{1}} \leqq \frac{m}{\rho \lambda_{1}}
$$

entfernt. Teilt man nun jede Seite des Einheitsquadrats in [ $\lambda]+1$ gleiche Teilstrecken und zieht durch die Teilpunkte Parallele zu den Koordinatenachsen, so wird das Einheitsquadrat in $([\lambda]+1)^{2}$ Teilquadrate ("Zellen") von dem Flächeninhalt $([\lambda]+1)^{-2}$ zerlegt. Offenbar muss nach dem soeben festgestellten jede Zelle, die einen Punkt $\alpha_{q}$ enthält, mit allen ihren Punkten um weniger als

$$
\frac{m}{\rho \lambda_{1}}+\frac{\sqrt{2}}{[\lambda]+1} \leqq \frac{192 \lambda}{\lambda_{1}}+\frac{\sqrt{2}}{[\lambda]+1}<\frac{4}{\lambda}
$$

von einer der Geraden (2) entfernt sein, Legt man folglich um jede der Geraden (2) als Mittellinie einen Parallelstreifen der Breite $\frac{8}{\lambda}$, so muss jede Zelle, die einen Punkt $\alpha_{q}$ enthält, mit allen ihren Punkten einem von diesen Streifen angehören. Nun ist offenbar erstens die Anzahl derjeniger von diesen Streifen, die das Einheitsquadrat durchsetzen, höchstens gleich $\sqrt{2}(\rho+2)$, und zweitens die Länge eines Streifens innerhalb des Einheitsquadrats höchstens gleich $\sqrt{2}$, so dass der ganze von den Streifen bedeckte Teil des Einheitsquadrats höchstens

$$
\sqrt{2}(p+2) \sqrt{2} \frac{8}{\lambda}=\frac{16}{\lambda}(p+2)
$$

zum Flächeninhalt hat; es gibt also höchstens

$$
([\lambda]+1)^{2} \frac{16}{\lambda}(\rho+2) \leqq 192 \lambda \rho
$$

Zellen, die Punkte $\sigma_{q}(q=0,1, \ldots, m)$ enthalten können; wegen $m=$ [192 \% p] ist $m+1>192 \lambda . \rho$, und folglich gibt es eine Zelle, die mindestens zwei von den Punkten $\alpha_{q}$ enthält. Indem man die Koordinaten. differenzen dieser beiden Punkte bildet, findet man

$$
\left|q \Theta_{1}-p\right|<\frac{1}{\lambda},\left|q \Theta_{2}-r\right|<\frac{1}{\lambda},
$$




$$
0<q \leqq m \leqq 192 \rho \lambda<192 \lambda \frac{\varepsilon}{1920} \sqrt{96 \lambda^{2}}<\varepsilon \lambda^{2},
$$

w. z. b. w

HILFSSATZ 2. Unter der Voraussetzung von Hilfssatz 1 gibt es eine Folge von natürlichen Zahlen $q_{1}, q_{2}, \ldots, q_{n}, \ldots$ mit folgenden Eigenschafteri:

$1^{0} . q_{n+1}>3 q_{n} \quad(n=1,2, \ldots)$.

$2^{0}$. Für jedes $\varepsilon>0$ und jedes $\lambda \geqslant \lambda_{0}=\lambda_{0}(\varepsilon)$ gibt es eine natürliche Zahl $n$ und ganze Zahlen $p_{k}, r_{k}(k=0,1, \ldots)$, die die Ungleichungen

$$
q_{n}<\varepsilon \lambda^{2},\left|q_{n+k} \Theta_{1}-p_{k}\right|<\frac{1}{\lambda},\left|q_{n+k} \Theta_{2}-r_{k}\right|<\frac{1}{\lambda} .(k=0,1, \ldots)
$$

erfïllen.

Beweis. - Für $\lambda \geqq 1$ sei $q=q(\lambda)$ die kleinste natürliche Zahl, welche zusammen mit zwei passend gewählten ganzen Zahlen $p, r$ die $\mathrm{Un}$ gleichungen

$$
\left|q \Theta_{1}-p\right|<\frac{1}{\lambda},\left|q \Theta_{2}-r\right|<\frac{1}{\lambda}
$$

erfüllt; es ist offenbar $q=q(\lambda)$ eine niemals abnehmende Funktion yon $\lambda_{i}$ ihre nacheinanderfolgenden Werte seien

$$
q_{1}, q_{2}, \ldots, q_{n}, \ldots
$$

Ist $\lambda$. genügend gross und $q_{n}=q(\lambda)$, so ist nach Hilfssatz $1 q_{n}<\eta \lambda_{\text {. }}$ wo $\eta$ eine beliebig vorgegebene positive Konstante bedeutet. Ist $q_{n}=$ $q\left(\lambda_{1}\right), q_{n+k}=q\left(\lambda_{1}\right), k \geqq 0$, so ist $\lambda_{1} \geqq \lambda$, und folglich, bei passend ge. wählten $p_{k}, r_{k}$

$$
\left|q_{n+k} \Theta_{1}-p_{k}\right|<\frac{1}{\lambda_{1}} \leqq \frac{1}{\lambda},\left|q_{n+k} \Theta_{2}-r_{k}\right|<\frac{1}{\lambda} \quad(k=0,1, \ldots) .
$$

Die Reihe (4) genügt somit der Forderung $2^{0}$, wenn $\eta=\varepsilon$ gewählt wird; um nun auch der Forderung $1^{0}$ Genüge $z u$ tun, verfahren wir folgenderweise.

Die Zahl $q^{n}$ der Reihe (4) heisse normal, wenn $q_{n+1}>3 q_{n}$ ist. Wir unterscheiden zwei Fälle.

A) Die Anzahl der normalen $q_{n}$ in (4) sei endlich. Für $n=n_{1}$ ist also $q_{n}$ nicht normal. Es sei allgemein $q_{n_{k+1}}$ die kleinste Zahl in (4), die grösser als $3 q_{n_{k}}$ ist $(k=1,2, \ldots) ;$ in der Folge

$$
q_{n}, q_{n_{2}}, \ldots, q_{n_{k}}, \ldots
$$

ist dann $q_{n_{k+1}}>3 q_{n_{k}}$. Ferner ist offenbar

$$
\frac{q_{n_{k+1}-1}}{q_{n_{k}}} \leqq 3 \text { und } \frac{q_{n_{k+1}}}{q_{n_{k+1}-1}} \leqq 3
$$

(letzteres weil $q_{n_{k+1}-1}$ nicht normal ist), woraus durch Multiplikation

folgt.

$$
q_{n_{k+1}} \leqq 9 q_{n_{k}}
$$

B) Die Anzahl der normalen $q_{n}$ in (4) sei unendlich. In diesem Fall wählen wir wieder eine Teilfolge aus (4), und namentlich so, dass wir zunächst alle normalen $q_{n}$ beibehalten. Es seien $q_{k}, q_{l}(k<l)$ zwei unmittelbar nacheinanderfolgende normale Glieder der Folge (4) $q_{l_{\mathrm{s}}}$ sei das grösste Glied, das $<\frac{1}{3} q_{l}$ ist; offenbar ist $q_{l_{1}} \geqq q_{k} ; q_{l_{1}}$ wird in die Teilfolge aufgenommen, aber alle etwaigen zwischen $q_{r_{1}}$ und $q_{t}$ liegenden Glieder von (4) werden gestrichen; ist $q_{t_{1}}>q_{k}$, so sei $q_{l_{2}}$ das grösste Glied von (4), das $<\frac{1}{3} q_{l_{1}}$ ist; offenbar ist $q_{l_{2}} \geqq q_{k} ; q_{l_{2}}$ wird in die Teilfolge aufgenommen, aber alle etwaigen zwischen $q_{l_{2}}$ und $q_{t_{1}}$ liegenden Glieder werden gestrichen; der Prozess wird fortgesetzt, bis einmal $q_{l_{i}}=q_{k}$ wird, was offenbar nach einer endlichen Anzahl von Schritten notwendig eintreten muss.

Bezeichnet man wieder mit $q_{n_{1}}, q_{n_{2}}, \ldots, q_{n_{k}}, \ldots$ die auf diese Weise gebildete Teilfolge von (4), so ist offenbar $q_{n_{k+1}}>3 q_{n_{k}}$, aber für $q_{n_{k}}<q_{s}<q_{n_{k+1}}$ stets $q_{s} \geqq \frac{1}{3} q_{n_{k+1}}$, da $q_{n_{k}}$ die grösste $\mathrm{Zahl}$ von (4) ist, die kleiner als $\frac{1}{3} q_{n_{k+1}}$ ausfällt.

Wir haben somit in allen Fällen eine Teilfolge (5) von (4) aufgéfunden, die den beiden Forderungen 1) $q_{n_{k+1}}>3 q_{n_{k}}$, 2) Für $q_{n_{k}}<q_{s}<$ $q_{n_{k+1}}$ ist $q_{n_{k+1}} \leqq 9 q_{s}$ genügt. Ist nun die Zahl $\lambda$. vorgegeben, so gibt es, wie wir schon wissen, ein $q_{s}$ in (4), so dass bei passend gewählten $p_{u}, r_{u}$

$$
q_{s}<\eta_{1} \lambda^{2},\left|q_{s+u} \Theta_{1}-p_{u}\right|<\frac{1}{\lambda},\left|q_{s+u} \Theta_{2}-r_{u}\right|<\frac{1}{\lambda} \quad(u=0,1, \ldots)
$$

ist; wird nun $k$ durch

$$
q_{n_{k}}<q_{s} \leqq q_{n_{k+1}}
$$


festgelegt ( $w$ à bei genügend grossem $\lambda$ evidenterweise stets möglich ist), so ist offenbar bei geeignet gewählten $p_{v}, r_{v}$ für $v=1,2, \ldots$

$$
\left|q_{n_{k+v}} \Theta_{1}-p_{v}\right|<\frac{1}{\lambda},\left|q_{n_{k+v}} \Theta_{2}-r_{v}\right|<\frac{1}{\lambda}
$$

und dabei ist

$$
q_{n_{k+1}} \leqq 9 q_{s}<9 r_{1} \lambda^{2}<\varepsilon \lambda_{2}^{2}
$$

wenn $\eta<\frac{\varepsilon}{9}$ gewählt wird. Die Folge (5) erfüllt demnach alle Forde* rungen von Hilfssatz 2, der somit bewiesen ist.

HILFSSATZ 3. Zu jeder positiven Zahl $\gamma$ gibt es eine andere positive Zahl $\mathrm{\Gamma}=\mathrm{\Gamma}(\gamma)$ mit folgender Eigenschaft. Haben die drei Zahlen q, $p_{1}, p_{2}$, keine gemeinsamen Teiler ausser 1 und ist für jede von $x_{1}=x_{2}=0$ verschiedene Lösung der Kongruenz

$$
q_{1} x_{1}+p_{2} x_{2}=0(\bmod q)
$$

$\rho^{2}=x_{1}{ }^{2}+x_{2}{ }^{2}>\gamma q$, so gibt es zu jeder ganzen Zahl gi zwei Zahlen $x_{1}$, $x_{2}$, die die Bedingungen

\section{erfüllen,}

$$
\left|x_{i}\right|<\mathrm{N} \sqrt{q}(i=1,2), \quad p_{1} x_{1}+p_{2} x_{2}=g(\bmod q)
$$

Dieser Hilfssatz bildet den auf $n=2$ bezogenen Spezialfall eines früher von mir bewiesenen allgemeinen Satzes ${ }^{1}$ ).

\section{3.}

Wir wenden uns nun zum Beweise des in 1. formulierten Hauptsatzes.

Um zunächst die Notwendigkeit der Voraussetzung zu begründen, nehmen wir $\Phi(\Theta)=0$ an, wählen ein $\varepsilon>0$, das später näher bestimmt werden soll, und betrachten die im Wortlaut von Hilfssatz 2 auftretende Zahlenfolge $q_{1}, q_{2}, \ldots q_{n}, \ldots$ Wegen $q_{2}>3 q_{1}$ fallen zwischen $\frac{1}{6 q_{1}}$ und $\frac{5}{6 q_{1}}$ notwendig zwei Punkte der Form $\frac{s_{2}}{q_{2}}, \frac{s_{2}+1}{q_{2}}$; wegen $q_{3}>3 q_{2}$ fallen zwischen $\frac{s_{2}+\frac{1}{6}}{q_{2}}$ und $\frac{s_{2}+\frac{5}{6}}{q_{2}}$ sicher zwei Punkte der Form $\frac{s_{3}}{q_{3}}, \frac{s_{3}+1}{q_{3}}$;

4) 1, c. $\left.{ }^{3}\right)$, S. 216, Hilfssatz III. indem man diese Schlussweise unbeschränkt fortsetzt, sieht man unmittelbar ein, dass die Intervalle

$$
\left(\frac{s_{n}}{q_{n}}, \frac{s_{n}+1}{q_{n}}\right)(n=1,2, \ldots)
$$

einen einzigen gemeinsamen Punkt $\beta$ enthalten, und dass für diesen

$$
\left|\beta-\frac{s}{q_{n}}\right|>\frac{1}{6 q_{n}}(n=1,2, \ldots, s \text { beliebig ganz })
$$

Nun sei, wenn möglich, $\urcorner(\theta, \beta)<+\infty$, also ein $\tau>0$ vorhanden, so dass die Ungleichung

$$
\left|x_{1} \theta_{1}+x_{2} \theta_{2}-y-\beta\right|<\frac{\tau_{1}}{\rho^{2}}\left(\rho=\sqrt{x_{1}{ }^{2}+x_{2}{ }^{2}}\right)
$$

mit beliebig grossen $\rho$ lösbar ist. Man setze im voranstehenden

$$
\varepsilon=\{6(\tau+2)\}^{-3} ;
$$

ferner wähle man eine Lösung von (7) mit

$$
\lambda=\rho \varepsilon^{-\frac{1}{3}}>\lambda_{0}(\varepsilon),
$$

wo $\lambda_{0}(\varepsilon)$ die in Hilfssatz 2 auftretende untere Schranke bedeutet. Nach der Eigenschaft $2^{n}$ der Zahfen $q_{n}$ ist dann bei geeigneten $n_{s} p_{n}, r_{n}$

$$
q_{n}<\varepsilon \lambda^{2}=\varepsilon^{\frac{1}{3}} \rho^{2},\left|q_{n} \Theta_{1}-p_{n}\right|<\frac{1}{\lambda},\left|q_{n} \Theta_{2}-r_{n}\right|<\frac{1}{\lambda} .
$$

also wegen (7), wenn $\hat{o}_{1}$ und $\hat{o}_{2}$ zwei Zahlen bedeuten, deren Absolutbeträge kleiner als 1 sind,

$$
\left|x_{1} \frac{p_{n}}{q_{n}}+\frac{x_{1} \delta_{1}}{\lambda q_{n}}+x_{2} \frac{r_{n}}{q_{n}}+\frac{x_{2} \delta_{2}}{\lambda q_{n}}-y-\beta\right|<\frac{\tau}{p^{2}} ;
$$

setzt $\operatorname{man} x_{1} p_{n}+x_{2} r_{n}-y q_{n}=s$, so folgt

$$
\left|\beta-\frac{s}{q_{n}}\right|<\frac{\tau}{\rho^{2}}+\frac{2 \rho}{\lambda q_{n}}<\frac{\tau \varepsilon^{\frac{1}{3}}}{q_{n}}+\frac{2 \varepsilon^{\frac{1}{3}}}{q_{n}}=\frac{1}{6 q_{n}} .
$$

was mit (6) in Widerspruch steht. Somit ist $\varphi(\Theta, \beta)=+\infty$, und die Notwendigkeit der Voraussetzung bewiesen.

Nun sei $\Phi(\Theta)>0$. Zunächst behaupte ich: $(A)$ es gibt eine positive 
Zahl $\tau=\tau(\Theta)$, so dass bei geeignet gewähltem, beliebig grossen 1. die Ungleichungen

(8)

$$
\left|q \Theta_{1}-p_{1}\right|<\frac{1}{\lambda},\left|q \Theta_{2}-p_{2}\right|<\frac{1}{\lambda}, 0<q \leqq \tau \lambda^{2}
$$

keine Lösungen in ganzen $q_{1} p_{1}, p_{2}$ haben.

In der Tat sei $\lambda>0,0<\varepsilon<\frac{1}{1500}$, und man setze

$$
\Lambda=\varepsilon \sqrt{\lambda}, \lambda_{1}=\frac{9 \sqrt{\lambda}}{\varepsilon},
$$

so dass

$$
\frac{\Lambda}{\lambda_{1}}=\frac{\varepsilon^{2}}{9}, \Lambda \lambda_{1}=9 \lambda
$$

wird. Wenn es ganze Zahlen $q, p_{1}, p_{2}$ gibt, die den Ungleichungen

$$
\left|q \Theta_{1}-p_{1}\right|<\frac{1}{\lambda_{1}},\left|q \Theta_{2}-p_{2}\right|<\frac{1}{\lambda_{1}}, 0<q<\varepsilon^{5} \lambda_{1}{ }^{2}
$$

genügen, so ist

$$
x_{1} \Theta_{1}+x_{2} \Theta_{2}=\frac{x_{1} p_{1}+x_{2} p_{2}}{q}+\frac{x_{1} \delta_{1}+x_{2} \partial_{2}}{q \lambda_{1}}\left(\left|\delta_{1}\right|<1,\left|\delta_{2}\right|<1\right)
$$

also bei passend gewählten ganzen $y_{,} s(0 \leqq s \leq q)$ und bei $\left|x_{1}\right| \leq . .1$, $\left|x_{2}\right| \leqq \Lambda$

$$
\left|x_{1} \Theta_{1}+x_{2} \Theta_{2}-y-\frac{s}{q}\right|<\frac{2 \Lambda}{q \lambda_{1}}, 0 \leqq x_{1} \Theta_{1}+x_{2} \Theta_{2}-y<1 .
$$

Durchlaufen nun die Variablen $x_{1}, x_{2}$ unabhängig voneinander die Zahlenreihe $0,1, \ldots,[\Lambda]$, so gehört offenbar jeder der $([\Lambda]+1)^{2}>\Lambda^{2}$ Punkte $x_{1} \Theta_{1}+x_{2} \Theta_{2}-y$ einer der Strecken

$$
\left(\frac{s}{q}-\frac{2 \Lambda}{q \lambda_{1}} \cdot \frac{s}{q}+\frac{2 \Lambda}{q \lambda_{1}}\right) \quad(0 \leqq s \leqq q)
$$

an ${ }^{5}$ ). Teilt man das Intervall $(0,1)$ in $[\lambda]+1$ gleiche Teilintervalle ("Zellen") der Länge $\frac{1}{[\lambda]+1}$, so kann jedes der Intervalle (9) höchstens mit

$$
\frac{4 \Lambda}{q \lambda_{1}}([\lambda]+1)+2
$$

5) Es wird dabei in üblicher Weise bei $s=0$ nur die rechte, bei $s=q$ nur die linke Hälfte des betreffenden Intervalls gerechnet und diese beiden Hälften werden zusammen als ein einziges. Intervall betrachtet.
Zellen gemeinsame Punkte haben. Es gibt also höchstens

$$
\begin{aligned}
& \frac{4 \Lambda}{\lambda_{1}}\left(\left[\lambda_{1}\right]+1\right)+2 q<\frac{8 \Lambda \lambda}{\lambda_{1}}+2 \varepsilon^{5} \lambda_{1}^{2}= \\
& =\frac{8}{9} \Lambda^{2}+162 \varepsilon^{3} \lambda=\frac{8}{9} \Lambda^{2}+162 \varepsilon \Lambda^{2}<\Lambda^{2}
\end{aligned}
$$

Zellen, die Punkte der Form $x_{1} \Theta_{1}+x_{2} \Theta_{3}-y$ enthalten können. Da nun die Anzahl dieser Punkte grösser als $\Lambda^{2}$ ist, muss eine Zelle mindestens zwei solche Punkte enthalten; und indem man ihre Differenz bildet, erhält man

$$
\begin{gathered}
x_{1} \Theta_{1}+x_{2} \Theta_{2}-y \mid<\frac{1}{\left[\lambda_{1}\right]+1}<\frac{1}{\lambda}, x_{1}{ }^{2}+x_{2}{ }^{2}>0, \\
\left|x_{1}\right| \leqq \Lambda=\varepsilon \sqrt{\eta_{1}}\left|x_{2}\right| \leqq \varepsilon \sqrt{\lambda_{.}} .
\end{gathered}
$$

Da dabei $\varepsilon$ beliebig klein und $\lambda$ beliebig (nur genügend grcss) ist, muss $\Phi(\Theta)=0$ sein, was der getroffenen Voraussetzung widerspricht. Damit ist unsere Behauptung $(\mathrm{A})$ bewiesen.

Es kann also $\lambda$. beliebig gross und derart gewählt werden, dass die kleinste natürliche Zahl $q$, die zusammen mit zwei ganzen Zahlen $p_{1}, p_{2}$ die beiden ersten Ungleichungen (8) befriedigt, grösser als $\tau \lambda^{2}$ ausfallen muss. Ich behaupte nun (B), dass jede von $x_{1}=x_{2}=0$ verschiedene Lösung der Kongruenz

(10)

der Ungleichung

$$
p_{1} x_{1}+p_{2} x_{2} \equiv 0(\bmod q)
$$

$$
p^{2}=x_{1}^{2}+x_{2}^{2}>\left(\frac{\tau}{200}\right)^{2} q
$$

genügen muss. Denn sei in der Tat $\gamma=\left(\frac{\tau}{200}\right)^{2}$.

$$
x_{1} p_{1}+x_{2} p_{2}+x_{3} q=0,0<\rho^{2} \leqq \gamma q .
$$

Die Variable $k$ durchlaufe die Zahlenreihe $1,2, \ldots, q$, und für jeden dieser Werte bestimme man zugehörige $l_{k}, m_{k}$, so dass

$$
0 \leqq \alpha_{k}=k \Theta_{1}-l_{k}<1,0 \leqq \beta_{k}=k \Theta_{2}-m_{k}<1
$$

ist. Wegen (8) und (11) ist $\left(\left|\hat{o}_{1}\right|<1,\left|\delta_{2}\right|<1\right)$

$$
\left|x_{1} \Theta_{1}+x_{2} \Theta_{2}+x_{3}\right|=\left|x_{1} \frac{p_{1}}{q}+\frac{x_{1} \delta_{1}}{q \lambda}+x_{2} \frac{p_{2}}{q}+\frac{x_{2} \delta_{2}}{q \lambda}+x_{3}\right|=
$$




$$
=\frac{1}{q \lambda_{1}}\left|x_{1} \hat{\partial}_{1}+x_{2} \hat{o}_{2}\right|<\frac{2 \sqrt{\gamma q}}{\lambda q}=\frac{2 \sqrt{\gamma}}{\lambda \sqrt{ } q}=\frac{0,01 \tau}{\lambda \sqrt{q}}
$$

und folglich für $1 \leqq k \leqq q$

$$
\begin{gathered}
\left|x_{1} \alpha_{k}+x_{2} \beta_{k}+l_{k} x_{1}+m_{k} x_{2}+k x_{3}\right|=k\left|x_{1} \Theta_{1}+x_{2} \Theta_{2}+x_{3}\right|< \\
<\frac{0,01 k \tau}{\lambda \sqrt{q}} \leqq 0,01 \tau \frac{\sqrt{q}}{\lambda} \equiv 0,01 \tau
\end{gathered}
$$

da bekanntlich $q \leqq \lambda^{2}$ ist. Es ist folglich jeder Punkt $\left(\alpha_{k}, \beta_{k}\right)$ des Einheitsqudrats um weniger als $\frac{0,01 \tau}{\rho}$ von einer der Geraden

$$
x_{1} \xi+x_{2} \eta+m=0 \text { ( } m \text { beliebig ganz) }
$$

entfernt. Legt man um jede Gerade (12), die das Einheitsquadrat durchsetzt, als Mittellinie einen Parallelstreifen der Breite

$$
\frac{0,02 \tau}{\rho}+\frac{2 \sqrt{2}}{\lambda}
$$

und zerlegt das Einheitsquadrat wie beim Beweise von Hilfssatz 1 in Zellen von der Seitenlänge $\frac{1}{[\lambda]+1}$, so muss jede Zelle, die einen Punkt $\left(\alpha_{k}, \beta_{k}\right)$ enthält, mit allen ihren Punkten einem dieser Streifen angehören. Da der von den Streifen eingenommene Flächenteil des Einheitsquadrats höchstens

$\sqrt{2}(\rho+2) \sqrt{2}\left(\frac{0,02 \tau}{\rho}+\frac{2 \sqrt{2}}{\lambda}\right)<6 \rho\left(\frac{0,02 \tau}{\rho}+\frac{4}{\lambda}\right)=0,12 \tau+24 \rho<<<24 \tau$

beträgt, können nicht mehr als ,

$$
0,24 \tau([\lambda]+1)^{2}<\tau \lambda^{2}<q
$$

Zellen yorhanden sein, die Punkte $\left(\alpha_{k}, \beta_{k}\right)$ enthalten, Da aber die Anzahl dieser Punkte gleich $q$ ist, muss es mindestens eine Zelle geben, die mindestens zwei solche Punkte enthält. Indem man die Differenzen der Koordinaten dieser Punkte bildet, findet man

$$
\left|k \Theta_{1}-r_{1}\right|<\frac{1}{\lambda},\left|k \Theta_{2}-r_{2}\right|<\frac{1}{\lambda}, 0<k<q,
$$

was nach der Definition von $q$ unmöglich ist; somit ist unsere Behauptung (B) bewiesen.
Da die Zahlen $q, p_{1}, p$, nach ihrer Definition keine gemeinsamen Teiler haben können, schliessen wir nach Hilfssatz 3 , dass für jede ganze Zahl $g$ zwei ganze Zahlen $x_{1}, x_{2}$ gefunden werden können, so dass

$$
\left|x_{i}\right| \leqq \Gamma_{1} q(i=1,2), p_{1} x_{1}+p_{2} x_{2} \equiv g(\bmod q)
$$

gilt; dabei ist $\Gamma$ eine positive Konstante, die nur von $\%$, also nur von $\Theta_{1}, \Theta_{2}$ (nicht von $q, p_{1}, p_{2}$ ) abhängt.

Ist nun $\beta$ eine beliebige reelle $Z$ ahl, so setze man

$$
[q \beta]=g, q \beta-g=\xi(0 \leqq \xi<1) .
$$

Werden zu diesem $g$ die Zahlen $x_{1}, x_{2}$ nach der soeben geschilderten Vorschrift festgelegt, so erhält man bei passend gewähltem $y$

$$
\begin{gathered}
\left|x_{1} \Theta_{1}+x_{2} \Theta_{2}-y-\beta\right|=\left|x_{1}\left(\frac{p_{1}}{q}+\frac{\hat{o}_{1}}{q \lambda}\right)+x_{2}\left(\frac{p_{2}}{q}+\frac{\hat{o}_{2}}{q \lambda}\right)-y-\frac{g}{q}-\frac{\xi}{q}\right|= \\
=\left|\frac{x_{1} \hat{o}_{1}+x_{2} \hat{o}_{2}}{q \lambda}-\frac{\xi}{q}\right|<\frac{2 \rho}{q \lambda}+\frac{1}{q} .
\end{gathered}
$$

und folglich wegen $p^{2} \leqq 2 \Gamma^{2} q, q \leqq \lambda^{2}$

$$
\left|x_{1} \Theta_{1}+x_{2} \Theta_{2}-y-\beta\right|<\frac{2 \rho}{q^{3 / 2}}+\frac{1}{q} \leqq \frac{I^{*}}{\rho^{2}} .
$$

wo $\Gamma^{*}>0$ nur von $\Theta_{1}, \Theta_{2}$ abhängt. Da dabei $q$ beliebig gross gewählt werden kann, ist auch $\rho$ beliebig gross, so dass $\varphi(\theta, \beta)$ tatsächlich endlich ist (im Ausnahmefall, wenn bei geeignet gewählten $x_{1}, x_{2}, y$ $x_{1} \Theta_{1}+x_{2} \Theta_{2}-y-\beta=0$ ausfällt, ist nach Definition $\left.\varphi(\Theta, \beta)=0\right)$. Die Voraussetzung $\Phi(\Theta)>0$ hat somit tatsächlich die Endlichkeit von $\varphi(\theta, \beta)$ für jedes $\beta$ zur Folge, und damit ist der in 1. angekündigte Satz vollständig bewiesen.

\section{4.}

Nach dem, was wir bewiesen haben, ist die Beziehung zwischen $\Phi(\Theta)$ und $\varphi(\theta, \beta)$ derjenigen zwischen $\varphi(\theta)$ und $\Phi(\Theta, \beta)$ vollständig analog. Zwischen den beiden Problemkreisen selbst bestehen jedoch sehr wesentliche Untẹsschiede, die wir noch kurz erörtern wollen.

Bei der früheren Fragestellung $\{\varphi(\Theta), \Phi(\Theta, \beta)\}$ war der "normale" Sachverhalt $(\varphi(\theta)>0, \phi(\theta, \beta)<+\infty)$ nur durch eine Nullmenge von Punkten $\left(\Theta_{1},\left(\Theta_{2}, \ldots, \Theta_{n}\right)\right.$ des $R^{n}$ vertreten; für fast alle Punkte hat man bekanntlich $\varphi(\theta)=0$ und folglich $\Phi(\Theta, \beta)=\omega$ bei geeignet gewähltem $\left.\beta^{6}\right)$.

i) K. Kap. V Satz 5. 
Deingegenüber lässt sich angesichts der neuen Fragestellung $\{\Phi(\Theta), \varphi(\theta, \beta)\}$ leicht beweisen, dass der normale Fall $(\Phi(\Theta))>0, \varphi(\Theta,(\beta)<+\infty)$ bei jedem $n$ für fast alle $\Theta$ vorliegt.

Bei der früheren Fragestellung bestand kein wesentlicher Unterschied zwischen dem ein-und dem mehrdimensionalen. Fall; bei der neuen Fragestellung ist ein solcher bekanntlich vorhanden; indem nämlich im eindimensionalen Fall für alle irrationalen $\Theta \Phi(\Theta)>0$ und folglich $\varphi(\theta, \beta)<+\infty$ gilt $^{7}$ ) ("normales" Verhalten), gibt es bei jedem $n>1$ ausser den trivialen $\theta$ mit linear abhängigen $\theta_{1},\left(\theta_{2}, \ldots, \theta_{n}\right.$ auch solche mit linear unabhängigen $\left(\Theta_{1}, \Theta_{2}, \ldots, \Theta_{n}\right.$ und mit $\left.\Phi\left(\omega_{)}\right)=0^{H}\right)$.

Endlich sei bemerkt, dass ich bei der früheren Fragestellung als Zusatz beweisen konnte: ist $\varphi(\theta)=0$, so ist für fast alle $\left.\beta \varphi(\Theta, \beta)=0^{\circ}\right)$. $\mathrm{Ob}$ ein analoger Zusatz auch bei der neuen Fragestellung gilt, weiss ich nicht; ich vermute, dass eine solche Behauptung im allgemeinen falsch wäre; auf jeden Fall scheint die hier entwickelte Methode keinen Anhaltspunkt für die Begründung eines derartigen Zusatzes zu liefern. (Eingegangen um 14, Mai 1936.)

7) K, Kap. III Satz 24 und Kap, VI Satz I.

8) K. Kap, V Satz 8.

9) K. Kap. VII Satz 2.

\section{An arithmetical theorem on linear forms.}

\author{
By
}

\author{
L. J. Mordell (Manchester).
}

Van der Corput ${ }^{1}$ ) has recently stated and proved the following exceedingly simple and general theorem:

"Ist $M$ eine im $n$-dimensionalen Raum liegende Menge vom Volumen $V>k_{1} k_{2} \ldots k_{n}\left(k_{1}>0, \ldots, k_{n}>0\right)$, und hat jedes zu $M$ gehörige Punktepaar $\left(x_{1}, \ldots x_{n}\right)$ und $\left(y_{1}, \ldots y_{n}\right)$ die Eigenschaft, dass der Punkt $\left(\frac{x_{1}-y_{1}}{k_{1}}, \ldots \frac{x_{n}-y_{n}^{\prime}}{k_{n}}\right)$ einer gewissen Menge $N$ angehört, dann enthält $N$ ausser dem Koordinatenursprung noch mindestens einen weiteren Gitterpunkt".

The proof tacitly assumes that $M$ is a simple set of points, i. e. that no point is reckoned more than once in calculating the volume. If $M$ is not a simple set, the theorem is not true as the origin may be the only lattice point in $N$. It then depends upon the nature of $M$ whether or not the result is trivial. As an illustration, I give a result on linear forms not included in his theorem, and so also not in Minkowski's famous theorem on homogeneous linear forms which it includes.

Let

$$
L_{r}(x)=\sum_{s=1}^{n} a_{r s} x_{s} \quad(r=1,2, \ldots n)
$$

1) Ván der Corput. Verallgemeinerung einer Mordellschen Beweismethode in der Geometrie der Zahlen, Acta Arithmetica, 1. (1935), 62-66. 